\title{
INQUIETAÇÕES E ESTRATÉGIAS DE ENFRENTAMENTO RELATADAS NAS RODAS DE TERAPIA COMUNITÁRIA INTEGRATIVA POR TRABALHADORES DE UM CENTRO DE SOCIOEDUCAÇÃO DO PARANÁ
}

\author{
PREOCUPACIONES Y ESTRATEGIAS DE ENFRENTAMIENTO REPORTADAS EN \\ LAS RUEDAS DE TERAPIA COMUNITARIA INTEGRADORA DE LOS \\ TRABAJADORES EN UN CENTRO DE SOCIODUCACIÓN DEL PARANÁ
}

\author{
CONCERNS AND COPING STRATEGIES REPORTED IN SESSIONS OF \\ INTEGRATIVE COMMUNITY THERAPY BY WORKERS IN A SOCIO- \\ EDUCATIONAL CENTER IN PARANÁ
}

\author{
Sandriane Aparecida Kalamar MARTINS ${ }^{1}$ \\ Sabrina STEFANELLO ${ }^{2}$ \\ Deivisson Vianna Dantas dos SANTOS $^{3}$ \\ Milene Zanoni da SILVA ${ }^{4}$
}

\begin{abstract}
RESUMO: Saúde Mental é uma importante questão de Saúde Pública quando relacionada a situações que envolvem o exercício do trabalho. A população deste estudo é formada de indivíduos trabalhadores de um CENSE/PR, ambiente fechado, prisional, que exige uma conduta de controle, vigilância e adequação frente às necessidades, favorecendo 0 desenvolvimento de sofrimentos. O objetivo do estudo foi identificar as principais inquietações e estratégias de enfrentamento relatadas nas rodas de TCI pelos trabalhadores. O estudo foi do tipo intervenção exploratório descritivo. Os dados foram submetidos a análise de conteúdo temática. Resultado das principais inquietações: trabalho 84,6\% (falta de reconhecimento), estresse 69,2\% (ansiedade e esgotamento); a principal estratégia de enfrentamento foi o fortalecimento e empoderamento pessoal 92,3\%. Conclui-se que intervenção com a TCI é eficaz na identificação de sofrimentos e estratégias de enfrentamento. É altamente recomendável gerar informações sobre a TCI para o cuidado e alívio do sofrimento de trabalhadores, colaborando à tomada de decisão na esfera pública.
\end{abstract}

PALAVRAS-CHAVE: Terapia comunitária integrativa. Socioeducação. Saúde do trabalhador. Promoção da saúde.

\footnotetext{
${ }^{1}$ Universidade Federal do Paraná (UFPR), Curitiba - PR - Brasil. Mestranda do Programa de Pós-Graduação em Saúde Coletiva. ORCID: https://orcid.org/0000-0002-6934-3621. E-mail:sandriane.martins@gmail.com

${ }^{2}$ Universidade Federal do Paraná (UFPR), Curitiba - PR - Brasil. Docente Permanente do Programa de PósGraduação em Saúde Coletiva. Doutorado em Ciências Médicas (UNICAMP). ORCID: https://orcid.org/00000002-9299-0405. E-mail: binastefanello@gmail.com

${ }^{3}$ Universidade Federal do Paraná (UFPR), Curitiba - PR - Brasil. Docente Permanente do Programa de PósGraduação em Saúde Coletiva. Doutorado em Saúde Coletiva (UNICAMP). ORCID: https://orcid.org/0000-00021198-1890. E-mail: deivianna@gmail.com

${ }^{4}$ Universidade Federal do Paraná (UFPR), Curitiba - PR - Brasil. Docente Permanente do Programa de PósGraduação em Saúde Coletiva. Doutorado em Saúde Coletiva (UEL). ORCID: https://orcid.org/0000-0002-11779668. E-mail: milenezanoni@gmail.com
} 
RESUMEN: La salud mental es un tema importante de salud pública cuando se trata de situaciones que involucran el ejercicio del trabajo. La población de este estudio está conformada por individuos que trabajan en un CENSE / PR, un ambiente carcelario cerrado, que requiere una conducción de control, vigilancia y adaptación a las necesidades, favoreciendo el desarrollo del sufrimiento. El objetivo del estudio fue identificar las principales preocupaciones y estrategias de afrontamiento reportadas en las ruedas TIC por los trabajadores. El estudio fue del tipo de intervención descriptiva exploratoria. Los datos se sometieron a análisis de contenido temático. Resultado de las principales preocupaciones: trabajo 84,6\% (falta de reconocimiento), estrés 69,2\% (ansiedad y agotamiento); la principal estrategia de afrontamiento fue el fortalecimiento y empoderamiento personal $92,3 \%$. Se concluye que la intervención con TIC es eficaz en la identificación del sufrimiento y estrategias de afrontamiento. Es muy recomendable generar información sobre las TIC para el cuidado y alivio del sufrimiento de los trabajadores, contribuyendo a la toma de decisiones en el ámbito público.

PALABRAS CLAVE: Terapia comunitaria integradora. Socioeducación. Salud del Trabajador. Promoción de la Salud.

ABSTRACT: Mental Health is an important Public Health issue when related to situations involving the exercise of work. The population of this study is formed by individuals working in a CENSE/PR, closed, prison environment, which requires a conduct of control, surveillance and adequacy to needs, favoring the development of suffering. The objective of the study was to identify the main worries and strategies of confrontation reported in the circles of ICT by the workers. The study was of the descriptive exploratory intervention type. The data were processed through thematic content analysis. Results of the main worries: work $84.6 \%$ (lack of recognition), stress $69.2 \%$ (anxiety and exhaustion); the main strategy of confrontation was personal strengthening and empowerment $92.3 \%$. It is concluded that intervention with the ICT is effective in identifying suffering and coping strategies. It is highly recommended to generate information about the ICT for the care and relief of suffering of workers, collaborating with decision making in the public sphere.

KEYWORDS: Integrative community therapy. Socio-education. Workers' health. Health promotion.

\section{Introdução}

A abrangência do tema Saúde Mental do trabalhador é por si só, bastante ampla. A Saúde Mental constitui-se numa importante questão de Saúde Pública, quando relacionada a situações que envolvem o exercício do trabalho, condição esta intrínseca a vida de um indivíduo ou grupo, enquanto ser social.

No contexto da vida humana faz-se importante compreender o exercício do trabalho, pois representa um dos aspectos que promovem a realização do indivíduo, mas que também confere um grau de responsabilidade frente a situações de desgastes físico, mental e emocional (MINAYO et al., 2010). 
De acordo com estudos, 160 milhões de indivíduos trabalhadores, no mundo, são afetados por doenças relacionadas ao trabalho; por exemplo, a depressão, prevalente nas mulheres, ocupa lugar de destaque, sendo considerada o quinto maior problema de Saúde Pública do mundo. No Brasil, os transtornos mentais comuns (TMC) ocupam a terceira maior causa das aposentadorias por invalidez dos trabalhadores (ROMERO, 2016; GAVIN et al., 2015).

A população deste estudo diz respeito a indivíduos trabalhadores que atuam em ambientes de controle e vigilância, no caso, os Centros de Socioeducação (CENSE). Esses espaços se caracterizam por serem unidades de atendimento que tem abrangência regional, vinculada à Secretaria de Estado da Família e Desenvolvimento Social (SEDS), que executam as medidas socioeducativas, ofertam programas à internação e/ou internação provisória aos adolescentes em conflito com a lei do Estado do Paraná em cumprimento de medida judicial (BRASIL, 2020b).

No âmbito paranaense, o atendimento das medidas de internação acontece na Secretaria de Justiça, Trabalho e Direitos Humanos (SEJU) por meio do Departamento de Atendimento Socioeducativo (DEASE) que administra 8 casas de Semiliberdade no Estado, que atendem crianças e adolescentes que possuem medida restritiva de liberdade: transição para meio aberto; e 19 CENSE que atendem crianças e adolescentes que possuem medida privativa de liberdade, internação como medida socioeducativa. Cada um destes Centros deve seguir os parâmetros da ação socioeducativa, os quais estão organizados pelos seguintes eixos estratégicos: suporte institucional e pedagógico; diversidade étnico-racial, de gênero e de orientação sexual; cultura, esporte e lazer; saúde; escola; profissionalização/trabalho/previdência; família e comunidade e segurança (BRASIL, 2020b).

Os CENSE dispõem de uma equipe de profissionais, que assumem a desafiadora tarefa de desenvolver atividades de relevância no processo de construção biopsicossocial com os adolescentes (BRASIL, 2018c). Assim a socioeducação e a saúde se entrelaçam frente aos desafios rotineiros, especialmente no que diz respeito a grupos sociais ou populações estigmatizadas (MOREIRA, 2019).

Devido às condições rotineiras de trabalho destes indivíduos, para com os/as adolescentes, que envolvem desde o auxílio na realização de atividades pedagógicas, manutenção da integridade física e psicológica, condutas de segurança, com uso de cadeados, portões de ferro; atividades externas vinculadas a saúde; atividades internas como lazer, banho de sol, etc.; estes indivíduos trabalhadores estão frequentemente expostos a diversas situações de desconforto, ameaças e agressividades (GRECO et al., 2013). 
Considera-se que exercer atividades de trabalho em espaços institucionais, tal como os CENSE, com características de ser fechado, prisional, exige destes trabalhadores uma conduta de controle, vigilância, atenção, segurança e adequação frente as necessidades adversas. Mediante tais circunstâncias é comum estes profissionais vivenciarem estados de tensão, pressão e estigma, interferindo nos aspectos afetivos e emocionais ocasionando o desenvolvimento de ansiedade, angústia, fadiga mental, depressão, exaustão, estresse, sofrimento psíquico, problemas nos relacionamentos familiares ou sociais, entre outros (BEZERRA et al., 2016).

A Terapia Comunitária Integrativa (TCI), é uma metodologia de intervenção. Criada pelo Prof. Dr. Adalberto Barreto, em 1987, com a intenção de solucionar as necessidades de saúde da comunidade do bairro Pirambu, Fortaleza - CE. Nasceu num contexto de precariedades em relação às condições da vida humana e a inúmeras deficiências, desde infraestrutura, segurança, emprego à atuação das políticas públicas (BARRETO, 2008; REIS, 2017).

A TCI, é um espaço comunitário onde as pessoas têm a oportunidade de falar sobre a sua história de vida, seus sentimentos, emoções, dor e conflitos de forma horizontal e circular. Transcende aos aspectos sociodemográficos e, caracteriza-se por ser uma ferramenta de construção de vínculos solidários, onde cada um torna-se terapeuta de si mesmo partilhando experiências de vida e sabedoria, todos se tornam corresponsáveis na busca de superações e soluções dos problemas cotidianos, o que propicia aos participantes experimentar a ajuda do grupo que oferece suporte e apoio (BARRETO, 2010).

Considerando a temática da Saúde Mental dos indivíduos trabalhadores da Socioeducação, baseando-se em algumas premissas como: o nível de transtornos mentais comuns entre os agentes socioeducativos (ROMERO et al., 2016) e o cenário da Socioeducação semelhante ao do sistema prisional, correlacionando o exercício do trabalho, estresse e adoecimento (GRECO et al., 2013), é pertinente investigar se as rodas de TCI são ferramentas capazes de identificar inquietações e estratégias de enfrentamento.

Sendo assim, o objetivo deste estudo foi identificar as principais inquietações e estratégias de enfrentamento, relatados na intervenção com as rodas de TCI, pelos trabalhadores de um CENSE do Paraná, capazes de gerar informações no cuidado, promoção da saúde e prevenção ou alívio de sofrimento, destes trabalhadores. 


\section{Percurso metodológico}

O presente estudo trata dos dados parciais que integram o projeto guarda-chuva "Manejos e práticas relacionadas às crises e ao sofrimento em saúde mental nos CENSE e casas de Semi Liberdade da Secretaria de Estado da Justiça, Cidadania e Direitos Humanos do Paraná”. Tal projeto guarda-chuva envolve um conjunto de dissertações de mestrado, dentre as quais a dissertação que originou essa pesquisa. Trata-se de uma pesquisa do tipo intervenção de caráter exploratório descritivo com abordagem qualitativa, que consiste na intervenção com as rodas de TCI, aqui denominadas por "intervenções".

Foram realizadas um total de 13 intervenções com as rodas de TCI, no período compreendido entre outubro de 2019 a fevereiro de 2020, prevalentemente nas quintas e sextasfeiras das 7 às 8 horas da manhã, no CENSE JMR, localizado no bairro Mercês em Curitiba/PR.

Cada intervenção com roda de TCI possuiu um número de participantes que oscilou entre um mínimo de três, e um máximo de onze, sendo que esses participantes pertenciam à população em estudo, composta por profissionais do quadro efetivo deste CENSE JMR, em um total de 45 indivíduos. Nessa população estavam incluídos os cargos/funções de agentes socioeducativos, equipe técnica, administrativo, motorista, equipe PROEDUSE $5 /$ professores e equipe terceirizada, sem filtros de exclusão para idade e tempo de serviço.

$\mathrm{O}$ acesso às rodas de TCI, enquanto aplicação de intervenção e participação na pesquisa, foi feito por convites intermediados pela Diretoria do CENSE JMR, na maior parte, e em alguns momentos, com a presença da pesquisadora. Além do convite verbal houve a fixação de convite impresso junto ao relógio ponto da instituição, num painel de avisos e na mesa do refeitório dos trabalhadores. O critério de inclusão foi definido em amostra por conveniência, e o critério de exclusão adotado foi o afastamento por licença para tratamento de saúde.

Todas as 13 intervenções aplicadas neste estudo foram conduzidas pela pesquisadora, que é terapeuta comunitária integrativa, capacitada por Polo Formador chancelado pela Associação Brasileira de Terapia Comunitária (ABRATECOM). Cada uma das intervenções foi gravada e transcrita para gerar os dados analisados neste estudo, sendo que os nomes dos participantes foram alterados para combinações de Letras, com o intuito de preservar o sigilo de identidade dos participantes da pesquisa, como determina a legislação sobre Pesquisas com Seres Humanos no Brasil (Resolução n ${ }^{0} 466$ - CNE/MS, 2012). Para realização deste estudo, a pesquisa foi aprovada pelo Comitê de Ética em Pesquisa da UFPR conforme número do CAAE:

${ }^{5}$ Programa de Educação nas Unidades dos CENSE, que oferta escolarização em todas as umidades de internação e internação provisória, sendo garantido o atendimento de ensino fundamental e médio na modalidade de Educação de Jovens e Adultos - EJA a todos os adolescentes (DEASE). 
02353018.3.0000.0102. Todos os participantes preencheram e assinaram o Termo de Consentimento Livre e Esclarecido - TCLE.

\section{Intervenção: as rodas de TCI desse estudo}

Cada roda de TCI foi considerada, nesse estudo, uma intervenção, e possui uma metodologia específica que, na prática, consiste em uma roda de conversa circular e horizontal, desenvolvida a partir de 6 etapas, descritas a seguir.

Acolhimento é a primeira etapa de uma roda de TCI e consiste no movimento de receber as pessoas, explicar a metodologia e apresentar as regras que norteiam a prática: silêncio e escuta ativa, a regra da cultura, não julgar e não inferir conselhos e falar de si, sempre em primeira pessoa.

Escolha do tema, ou a inquietação é a segunda etapa de uma roda de TCI, como momento de compartilhar a inquietude ou sofrimento com o grupo: a pessoa diz seu nome e em poucas palavras procura nomear o que sente, apresentando de três a cinco breves relatos, dependendo do grupo. Nessa etapa também é possível abrir um espaço de fala à identificação e justificativa do que ressoou na sua própria história de vida; feita essa fala, passa-se à votação pela história que mais ressoou no grupo e, a partir desse procedimento, vem a decisão pelo tema a ser aprofundado.

A contextualização é a terceira etapa de uma intervenção pela roda de TCI, e consiste do momento em que o protagonista que trouxe o tema escolhido pode falar mais da sua vivência, e nesse momento todos os participantes podem fazer perguntas para o protagonista, com o objetivo de ampliar a reflexão e compreensão do sofrimento da situação.

Partilha de experiências/problematização, onde a terapeuta elabora uma pergunta pertinente ao tema que emergiu na roda e lança no grupo para que todos, ou aqueles que sentirem-se conectados, possam responder, trazendo suas respostas e ferramentas, enquanto estratégias de superação.

Encaminha-se, assim, para o encerramento, que é um momento onde a terapeuta convida a comunidade ou grupo a receber o agradecimento que fará aos integrantes e ao protagonista, enfatizando que todas as histórias são importantes. É no encerramento que a terapeuta convida todos a se manifestarem, verbalizando aquilo que mais o tocou, ou o que está levando como aprendizado, dessa roda de TCI. 
Por fim, a apreciação da terapia consiste na avalição, de acordo com um roteiro de apoio, do processo de condução da roda de TCI, que nesse estudo foi conduzida apenas pela pesquisadora, como terapeuta.

Foi o roteiro de apoio da apreciação da condução da intervenção, conforme sistematizado na metodologia da TCI, que serviu de base para gerar as informações tabuladas neste estudo (BARRETO, 2008; REIS, 2017).

\section{Tratamento dos resultados}

Os dados foram coletados por meio de questionário semiestruturado acerca das variáveis sociodemográficas, e pelo instrumento de apreciação da TCI.

Todos os dados foram planilhados, gerando um banco de dados que foi submetido a tratamento e análise temática de conteúdo.

\section{Resultados}

\section{Caracterização sociodemográfica}

Um total de 37 trabalhadores integrou o universo da pesquisa, sendo que a distribuição de gênero foi de 24 mulheres (64,9\%) e 13 homens (35,1\%); quanto à orientação sexual, 94,6\% manifestou a heterossexualidade como opção. Todas as características pessoais de identificação autorreferidas foram registradas e estão descritas na Tabela 1, juntamente com o apontamento dos participantes, em relação à função exercida no quadro efetivo do CENSE JMR.

Tabela 1 - Distribuição de dados sociodemográficos dos integrantes que participaram da pesquisa $(n=37)$

\begin{tabular}{lcc}
\hline Variáveis & N & $\mathbf{\%}$ \\
\hline Sexo & & \\
\hline Feminino & 24 & 64,9 \\
\hline Masculino & 13 & 35,1 \\
\hline Orientação sexual & 35 & 94,6 \\
\hline Heterossexual & 1 & 2,7 \\
\hline Homossexual & 1 & 2,7 \\
\hline Outros & & \\
\hline Quanto à cor da pele/etnia: & 27 & 73,0 \\
\hline Branco(a)
\end{tabular}




\begin{tabular}{|c|c|c|}
\hline Pardo(a) & 9 & 24,3 \\
\hline Amarelo(a) & 1 & 2,7 \\
\hline \multicolumn{3}{|l|}{ Situação conjugal/estado civil } \\
\hline Solteiro (a) & 9 & 24,3 \\
\hline Casado (a) & 21 & 56,8 \\
\hline Separado/divorciado (a) & 4 & 10,8 \\
\hline União consensual & 3 & 8,1 \\
\hline \multicolumn{3}{|l|}{ Escolaridade } \\
\hline 2॰ Grau incompleto & 1 & 2,7 \\
\hline 2。 Grau completo & 2 & 5,4 \\
\hline 3० Grau incompleto & 2 & 5,4 \\
\hline 3० Grau completo & 14 & 37,8 \\
\hline Pós-Graduação & 18 & 48,6 \\
\hline \multicolumn{3}{|l|}{ Renda familiar } \\
\hline Até 5 salários mínimos & 14 & 37,8 \\
\hline De 6 a 10 salários mínimos & 17 & 45,9 \\
\hline Acima de 10 salários mínimos & 6 & 16,2 \\
\hline \multicolumn{3}{|l|}{ Prática alguma atividade física } \\
\hline Não & 10 & 27,0 \\
\hline Sim, esporadicamente & 8 & 21,6 \\
\hline Sim, com frequência & 19 & 51,4 \\
\hline $\begin{array}{l}\text { Condições de saúde autorreferida: depressão e/ou } \\
\text { ansiedade }\end{array}$ & 13 & 35,1 \\
\hline
\end{tabular}

Uso de medicamentos

\begin{tabular}{lccc}
\hline Não & 19 & 51,4 \\
\hline Sim & 18 & 48,6
\end{tabular}

Vínculo que considera importante em momentos de dificuldades

\begin{tabular}{lccc}
\hline Família & 34 & 91,9 \\
\hline Amigos & 16 & 43,2 \\
\hline Comunidade & 2 & 5,4 \\
\hline Trabalho & 2 & 5,4 \\
\hline Serviços de saúde & 2 & 5,4 \\
\hline
\end{tabular}

Função no CENSE

\begin{tabular}{lccc}
\hline Agentes de Segurança Socioeducativa (ASSE) & 22 & 59,5 \\
\hline Equipe Técnica & 5 & 13,5 \\
\hline Administrativo & 1 & 2,7 \\
\hline Equipe Proeduse/Professores & 8 & 21,6 \\
\hline Equipe terceirizada & 1 & 2,7 \\
\hline & $\mathbf{n}$ & anos
\end{tabular}




\begin{tabular}{lcc} 
Idade média & 34 & 44,0 \\
\hline Tempo Médio de atuação no CENSE & 23 & 10,8 \\
\hline Tempo Médio de atuação no CENSE JMR & 23 & 8,4 \\
\hline
\end{tabular}

Fonte: Dados da pesquisa (2019-2020)

\section{Caracterização dos temas (inquietações) e das estratégias de enfrentamento relatados na intervenção com a TCI}

Na Tabela 2 estão apresentados, em ordem de frequência de distribuição percentual, os temas universais citados nas 13 intervenções com rodas de TCI, objetos desta pesquisa. $\mathrm{Na}$ referida Tabela, encontram-se em destaque negrito os temas universais, e sublinhadas, as expressões que permearam a fala dos participantes, cada uma delas atribuídas aos temas universais.

Tabela 2 - Descrição dos temas universais citados nas rodas de TCI realizadas no CENSE

JMR, Paraná, 2020 (n=13)

\begin{tabular}{l|c|c}
\hline Temas Universais & $\mathbf{n}$ & $\%$ \\
\hline Trabalho (Desrespeito, indisciplina, falta de reconhecimento, desemprego, & 11 & 84,6 \\
insatisfação, insegurança, agressão física/verbal e moral, dificuldades & & \\
financeiras...) & & \\
\hline Estresse (angústia, medo, ansiedade, insônia, nervosismo, mágoa, raiva, & 9 & 69,2 \\
vingança, desânimo, desespero, esgotamento...) & & \\
\hline Conflitos familiares (marido e mulher, relacionamentos íntimos, separação, \\
pais, filhos, irmãos, avós, netos, traição, ciúmes...) & 2 & 15,4 \\
\hline $\begin{array}{l}\text { Violência (adolescentes, criança, policial, sexual, assalto, intrafamiliar } \\
\text { contra a mulher/homem, idoso, homicidio, gangues, exploração sexual, } \\
\text { pedofilia...) }\end{array}$ & 1 & 7,7 \\
\hline
\end{tabular}

Fonte: Dados da pesquisa (2019-2020)

As estratégias de enfrentamento relatadas durante as intervenções com as rodas de TCI encontram-se apresentadas de acordo com a frequência de distribuição percentual na Tabela 3. 
Tabela 3- Descrição das estratégias de enfrentamento relatadas durante as rodas de TCI realizadas no CENSE JMR, Curitiba, 2020 (n=13)

\begin{tabular}{l|c|c}
\hline Estratégias de enfrentamento & $\mathbf{n}$ & $\mathbf{\%}$ \\
\hline $\begin{array}{l}\text { Fortalecimento/Empoderamento pessoal: capacidade de apropriar-se do } \\
\text { seu poder de agir }\end{array}$ & 12 & 92,3 \\
\hline Participar da terapia comunitária & 3 & 23,1 \\
\hline Autocuidado (busca de recursos na cultura) & 3 & 23,1 \\
\hline Busca de ajuda profissional e ações de cidadania (serviços públicos) & 2 & 15,4 \\
\hline Busca de ajuda religiosa ou espiritual & 2 & 15,4 \\
\hline Busca de redes solidárias (amigos, vizinhos, associações, grupos de & 2 & 15,4 \\
autoajuda) & & \\
\hline Cuidar do relacionamento com a família & 2 & 15,4 \\
\hline
\end{tabular}

Fonte: Dados da pesquisa (2019-2020)

\section{Discussão}

Com base no conceito e na estrutura metodológica da ferramenta de intervenção utilizada a TCI, foi possível extrair das rodas de TCI, fazendo uso do roteiro de apoio, conteúdos que possibilitaram mapear os temas universais (inquietações) que retratam o sofrimento humano, assim como as estratégias de enfrentamento dos participantes.

Faz-se importante destacar que a população alvo deste estudo diz respeito a trabalhadores de um CENSE do Paraná, que conforme Tabela 1, apresenta elevado percentual de trabalhadores com pós-graduação $(48,6 \%)$, sendo que a maioria dos trabalhadores ocupam a função de agentes de segurança socioeducativa (59,5\%), com idade média de 44 anos e tempo médio de atuação mais de dez anos dentro do sistema da socioeducação.

Verifica-se, que na Tabela 2, os temas universais relatados com maior representatividade foram dois. Primeiramente, o trabalho, que aparece com uma frequência de $84,6 \%$. As expressões que permearam a fala dos participantes em relação ao tema foram a falta de reconhecimento e de respeito, conforme em destaque sublinhado. Este achado também possibilita uma correlação com a Tabela 1 e pressupõem coerência com o item que trata do "vínculo que considera importante em momentos de dificuldades", em que o trabalho $(5,4 \%)$, apresenta baixo vínculo de apoio.

Em segundo, vem o estresse (69,2\%), com manifestações expressivas dos participantes de ansiedade, angústia e esgotamento. Vale destacar que ambientes de trabalho como a socioeducação com intensas demandas emocionais, psicológicas, estão associados aos maiores riscos à saúde (GRECO et al., 2013). 
Já na Tabela 3, quanto a estratégia de enfrentamento, predomina com 92,3\% o Fortalecimento/Empoderamento pessoal: a capacidade de apropriar-se do seu poder de agir. Esse dado também possibilita correlacionar com itens da Tabela 1, considerando que 51,4\% praticam atividade física com frequência, e que a família foi apresentada como importante base de apoio em 91,9\% das respostas, seguida dos amigos em 43,2\%.

Diante do exposto, observa-se que a TCI é uma importante tecnologia psicossocial de intervenção para avaliação, diagnóstico, promoção e cuidado da saúde do trabalhador.

\section{Considerações}

Este estudo atingiu o objetivo proposto constatando que a intervenção, com as rodas de TCI, é eficaz na identificação das inquietações relacionadas ao sofrimento dos trabalhadores, assim como na identificação das estratégias de enfrentamento utilizadas pelos trabalhadores de um CENSE do Paraná.

Os achados indicam preocupante realidade de sofrimento e estresse em relação ao exercício do trabalho desses profissionais. Portanto, fica evidente a necessidade de se buscar estratégias de cuidado para o contexto dos trabalhadores dentro da socioeducação.

Ainda, é imprescindível gerar informações sobre a TCI para o cuidado, promoção da saúde e prevenção ou alívio do sofrimento de trabalhadores e para a tomada de decisão na esfera pública, uma vez que desde 2008 está inserida no contexto do Sistema Único de Saúde (SUS), como prática integrativa e complementar de cuidado em saúde mental (ANDRADE, 2009).

Por fim, recomenda-se possibilitar a implantação e o acesso a práticas de intervenção com as rodas de TCI, uma vez que a metodologia promove a expressão, a troca de experiências e saberes, repercute na construção da autonomia e no fortalecimento de vínculos solidários, gerando empoderamento individual e coletivo na perspectiva de qualificar o exercício do viver.

AGRADECIMENTOS: O projeto teve solicitação da Secretária de Estado da Justiça, Trabalho e Direitos Humanos (SEJU), da divisão de saúde do Departamento de Atendimento Socioeducativo (DEASE) e apoio da Direção do CENSE Joana Miguel Richa (CENSE JMR).

\section{REFERÊNCIAS}

ANDRADE, L. O. M. et al. (Orgs.). Ministério da Saúde. Departamento de Atenção Básica. O SUS e a Terapia Comunitária. Fortaleza: Ministério da Saúde, 2009. 
BARRETO, A. P. Terapia comunitária: passo a passo. 3. ed. rev. ampl. Fortaleza: Gráfica LCR, 2008.

BARRETO, A. P. Terapia comunitária: passo a passo. 4. ed. Fortaleza: LCR, 2010.

BEZERRA C. M et al. Sofrimento psíquico e estresse no trabalho de agentes penitenciários: uma revisão da literatura. Ciência \& Saúde Coletiva, v. 21, n. 7, p. 2135-2146, 2016.

BRASIL. Cadernos de socioeducação: gestão pública do sistema socioeducativo. Curitiba, PR: Secretaria da Justiça, Trabalho e Direitos Humanos, 2018. Disponível em:

http://www.justica.pr.gov.br/sites/default/arquivos_restritos/files/migrados/File/CadernoGesta o__.pdf. Acesso em: 12 abr. 2020.

BRASIL. Socioeducação. Curitiba, PR: SEJUF, DEASE, 2020.Disponível em: http://www.justica.pr.gov.br/Pagina/Socioeducacao-0. Acesso em: 7 abr. 2020.

GAVIN, R. S. et al. Associação entre depressão, estresse, ansiedade e uso de álcool entre servidores públicos. SMAD, Revista Electrónica em Salud Mental, Alcohol y Drogas, v. 11, n. 1, p. 2-9, jan./mar. 2015.

GRECO, P. B. T. et al. Estresse no trabalho em agentes dos centros de atendimento socioeducativo do Rio Grande do Sul. Rev. Gaúcha Enferm, v. 34, n. 1, p. 94-103, 2013.

MINAYO, M. C. S. Introdução. In: MINAYO, M. C. S.; ASSIS, S. G.; SOUZA, E. R. (Org.). Avaliação por triangulação de métodos: abordagem de programas sociais. Rio de Janeiro: Fiocruz, p. 19-51, 2010.

MOREIRA, I. A. W. Políticas educacionais e desigualdade social no brasil: desafios à inclusão social de adolescentes autores de atos infracionais. Orientador: Telmo Marcon. 2019. 451 f. Tese (Doutorado em Educação) - Universidade de Passo Fundo, Passo Fundo, RS, 2019. Disponível em: http://tede.upf.br:8080/jspui/handle/tede/1884. Acesso em: 1 abr. 2020.

REIS, M. L. A. Quando me encontrei voei: o significado da capacitação em Terapia Comunitária Integrativa. Porto Alegre: CAIFCOM Editora, 2017.

ROMERO, D. L et al. Transtornos mentais comuns em educadores sociais. J. Bras. Psiquiatr., v. 65, n. 4, p. 322-329, 2016.

SILVA, D. C et al. Tecnologias leve e cuidado em enfermagem. Esc. Anna Nery Ver Enferm., v. 12, n. 2, p. 291-298, jun. 2008. 


\section{Como referenciar este artigo}

MARTINS, S. A. K.; STEFANELlO, S.; SANTOS, D. V. D. dos; SILVA, M. Z. da. Inquietações e estratégias de enfrentamento relatadas nas rodas de terapia comunitária integrativa por trabalhadores de um centro de socioeducação do Paraná. Temas em Educ. e Saúde, Araraquara, v. 16, n. esp. 1, p. 409-421, set., 2020. e-ISSN 2526-3471. DOI: https://doi.org/10.26673/tes.v16iesp.1.14320

Submetido em: 20/05/2020

Revisões requeridas: $30 / 05 / 2020$

Aprovado em: 25/08/2020

Publicado em: 30/09/2020 